\title{
Clinical and Radiological Comparison of Semirigid (WavefleX) and Rigid System for the Lumbar Spine
}

\author{
Do-Keun Kim${ }^{1}$, Hyunkeun $\mathrm{Lim}^{2}$, Dae Cheol Rim ${ }^{3}$, Chang Hyun $\mathrm{Oh}^{4}$ \\ Departments of ${ }^{I}$ Neurosurgery and ${ }^{2}$ Anesthesiology \& Pain Medicine, Inha University School of Medicine, Incheon, \\ ${ }^{3}$ Department of Neurosurgery, Kim Young Soo Spine \& Joint Hospital, Seoul, \\ ${ }^{4}$ Department of Neurosurgery, Cham Teun Teun Reserarch Institute, Seoul, Korea
}

\begin{abstract}
Objective: Spinal fusion operation is an effective treatment in the spinal pathology, but it could change the physiological distribution of load at the instrumented and adjacent segments. This retrospective study compared the radiological and clinical outcomes of patients undergoing lumbar fusion with semirigid rods versus rigid rods system.

Methods: Using transpedicular fixation and posterior lumbar interbody fusion at the level of L4/L5, 20 patients were treated with semirigid rods (WavefleX, SR group), and 20 patients with rigid rods (titanium, RR group). Clinical and radiological outcomes were evaluated, including visual analog score for lower back pain and leg pain, Prolo functional and economic scores, statues of implanted instruments, fusion rate, and complications during 24-month follow-up.

Results: Clinical scores were significantly improved until postoperative 24-month follow-up as compared with preoperative scores in both groups $(p<0.05)$, with similar levels of improvement observed at the same time points postoperatively between the 2 groups. Prolo economic scores were significantly improved in SR group compared to RR until 12 months, but this improvement became similar after 18 months. The overall fusion rate was $94.1 \%$ until the 24 -month follow-up for both groups. No significant complication was observed in both groups.

Conclusion: The results of the present study indicate that semirigid rods system with posterior lumbar interbody fusion showed similar clinical and radiological result with rigid rods system until 2 years after instrumentation. The WavefleX rods system, as a semirigid rods with unique characteristics, may be an effective alternative treatment for patients in lumbar fusion.
\end{abstract}

Key Words: Spinal fusion $\cdot$ Semirigid instruments $\cdot$ WavefleX $\cdot$ Outcome $\cdot$ Fusion

\section{INTRODUCTION}

Fusion is a widespread and accepted treatment for painful degenerative conditions of the lumbar spine ${ }^{11)}$. Overall, the outcome after fusion appears to be quite inconsistent: a systematic review of mainly retrospective case series reported that satisfactory clinical outcomes ranged from just $16 \%$ to as high as $95 \%$, with an average of around $68 \%{ }^{22}$. Rigid posterior fixation is one of the most popular techniques to achieve a higher fusion rate, although, the benefits from this higher fusion rate have not yet been identified in clinical outcomes ${ }^{3,4,8,14)}$. Various adverse effects of rigid fixation, including increased intradiscal pressure, increased facet loading of the adjacent

- Received: January 6, 2016 • Revised: March 15, 2016

- Accepted: April 6, 2016

Corresponding Author: Dae Cheol Rim

Department of Neurosurgery, Kim Young Soo Spine \& Joint Hospital,

15, Eonju-ro 174-gil, Gangnam-gu, Seoul 06017, Korea

Tel: +82-2-1577-5075, Fax: +82-2-3447-0015

E-mail: nsrimok@gmail.com

@This is an open access article distributed under the terms of the Creative Commons Attribution Non-Commercial License (http://creativecommons.org/licenses/by-nc/4.0/) which permits unrestricted non-commercial use, distribution, and reproduction in any medium, provided the original work is properly cited. segments, and a stress shielding effect on bone graft materials, had been reported ${ }^{5,17}$. The bony fusion and the effects of lumbar arthrodesis on adjacent segments could vary according to the surgical technique used for arthrodesis. But, there have been few reports on the differences in the effects of different arthrodesis techniques on adjacent segment disease. In a study using finite element analysis ${ }^{14)}, 2$ arthrodesis models were analyzed and compared; semirigid fixation model combined with posterior lumbar interbody fusion and rigid fixation model combined with posterior lumbar interbody fusion. The rigid fixation model showed the greater amount of stress, with increased intervertebral disc pressure and contact force of the facet joints of both upper and lower adjacent segments compare to the semirigid fixation model. However, there are no clinical studies as well as in vivo biomechanical studies were present after this finite element analysis ${ }^{14)}$. The present retrospective study compared the clinical and radiological outcomes of patients undergoing lumbar fusion with semirigid rods versus rigid rods.

\section{MATERIALS AND METHODS}

Forty patients were retrospectively reviewed in this study between January 2012 and October 2013 at the single spine 
hospital, Korea (Table 1). These patients were treated by transpedicular fixation and lumbar fusion: 20 patients (semirigid rods, SR group) were treated with WavefleX rods system (Medyssey Co., Uijeongbu, Korea; Fig. 1) and 20 patients (rigid rods, RR group) were treated with titanium rods system. Patients were enrolled in the study if they presented with single-level, lumbar degenerative disease at the level of L4/L5, including lumbar disc herniation with segmental instability, lumbar spondylotic stenosis with segmental instability, or lowgrade degenerative lumbar spondylolisthesis. Lumbar segmental instability was defined as sagittal translation of $3 \mathrm{~mm}$ or more and sagittal angulations of $10^{\circ}$ or more on dynamic flexion-extension films ${ }^{12,20}$. Patient inclusion criteria were persistent lower back pain and/or leg pain after an unsuccessful conservative therapy over a period of at least 6 months, and willingness to take part in follow-up procedures. The exclusive criteria included multilevel lumbar degenerative disease; a history of an earlier back operation; an extraspinal cause of lower back pain or leg pain; spinal infections and tumors; inflammatory arthritis; or metabolic bone disease.

All surgeries were conducted by the single spine surgeon (DCL). All cases underwent single-level posterior lumbar interbody fusion at the level of L4/L5. A midline skin incision was created, the fascia was incised, and the paravertebral muscles were dissected from the spinous processes. Fluoroscopy was used to determine the operative level. Bilateral pedicle screws were first inserted. Posterior bony decompression was performed at the appropriate level. The locally harvested bone was later used as interbody fusion graft material. A discectomy was performed and the cartilaginous material was removed carefully from the endplates using an endplate scraper. Care was taken to avoid damage to the subchondral endplates. Distraction of the disc space was then carried out and supported by temporarily locking a titanium rod to the transpedicular screws in both groups. Excess autogenous bony particles were placed into the intervertebral space. Intervertebral polyether ether ketone (PEEK) cage, packed with local autogenous bones,

Table 1. Demographic data of retrospective series

\begin{tabular}{lccc}
\hline \hline Variable & SR group & RR group & p-value \\
\hline Age (yr) & $66.6 \pm 13.2$ & $64.6 \pm 12.7$ & 0.637 \\
Male sex ratio & $45 \%$ & $40 \%$ & 0.749 \\
Diagnosis & & & 1.000 \\
$\quad$ Disc disease & 10 & 10 & \\
$\quad$ Spinal stenosis & 10 & 10 & \\
Follow-up & & & \\
$\quad$ Preoperative & 20 & 20 & 1.000 \\
Postoperative 6 months & 20 & 20 & 1.000 \\
Postoperative 12 months & 20 & 20 & 1.000 \\
Postoperative 18 months & 19 & 18 & 0.548 \\
Postoperative 24 months & 17 & 17 & 1.000 \\
\hline
\end{tabular}

$\mathrm{SR}$, semirigid rod; $\mathrm{RR}$, rigid rod. was inserted into the intervertebral space for support and fusion for both groups. In the SR group, after placement of the cages, the titanium rods were placed by semirigid rods. Final compression was performed using a compressor device in the pedicle screw-rod connection system to provide compression to the bone graft and the lumbar lordosis. In each patient, adequacy of the nerve root decompression was checked before the skin was sutured. During the first 3-month postsurgery, the patients were immobilized in a lumbar spinal brace whenever they were out of bed. Patients were encouraged to return to work as soon as they felt capable.

Clinical and radiological outcomes were measured preoperatively and postoperatively. Clinical outcomes were determined using the visual analog score for lower back pain (VAS-B) and leg pain (VAS-L), and Prolo Scores. Prolo scores is a 10point scale consisting of only two questions evaluating the functional and economic status of the patient ${ }^{11)}$. Prolo economic scores (PESs) consisted as complete invalidity (1 point), no gainful occupation, including ability to do housework, or continue retirement activities (2 points), able to work, but not at previous occupation; able to perform housework and retirement activities ( 3 points), working at previous occupation parttime or limited status (4 points), and able to work at previous occupation with no restrictions of any kind (5 points). Prolo functional scores (PFSs) consisted as total incapacity (postoperative: worse than prior to operation, 1 point), difficulty in walking, needing a cane or crutch or persistent moderate motor weakness in upper limb (able to perform tasks of daily living, 2 points), slight difficulty in walking, but without help; slight motor weakness in upper limb, moderate pain, persistent paresthesia (3 points), no difficulty in walking, no motor weakness in upper limb, no pain but persistent paresthesia (4 points), and no difficulty in walking, no motor weakness in upper limb, no pain, no paresthesia, able to perform sports activities (5 points). It can be described as an outcome disability tool originally designed for use as an outcome measurement for patients in whom underwent posterior lumbar interbody fusions, and measured economic and functional status of the patient before and after treatment. The outcome scores for the patients were

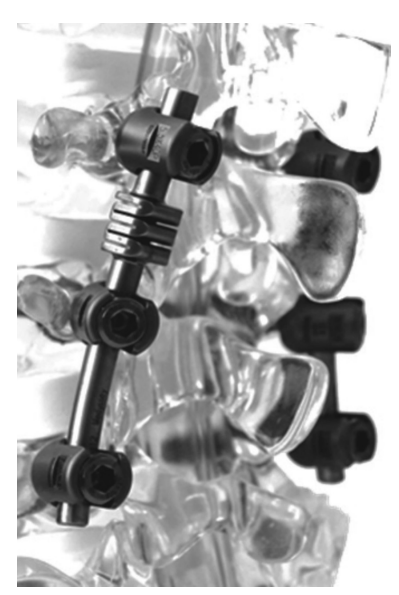

Fig. 1. Image of WavefleX rods system (Medyssey Co., Uijeongbu, Korea) as a semirigid rods. 
collected preoperatively and at $6,12,18$, and 24 months postoperatively.

All patients underwent preoperative lumbar dynamic radiographs and magnetic resonance imaging scanning. Sagittal dynamic angulations (adjacent level, L3/L4 and L5/S1) preoperative dynamic flexion-extension films were measured, and follow-up dynamic radiographs were taken at $6,12,18$, and 24 months postoperatively (Fig. 2). Radiographic assessments included the status of the implanted instruments, fusion rate and complications. Fusion was defined as a demonstration of confluent bony trabeculation across the intervertebral space
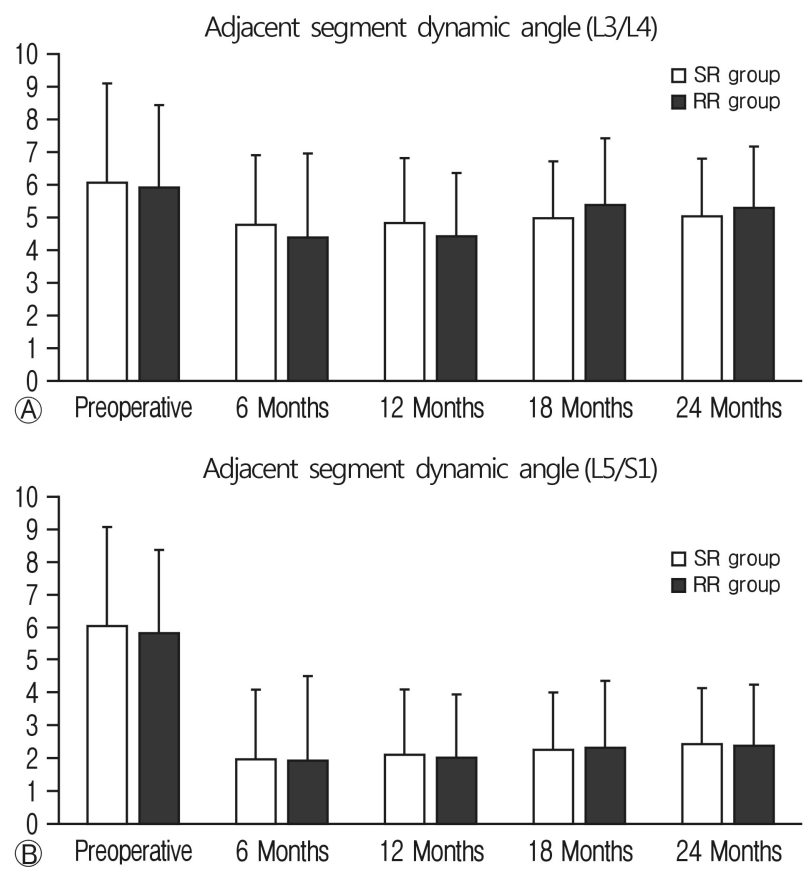

Fig. 2. Postroperative segmental dynamic angle changes at L3/ 4 (A) and L5/S1 (B) level.
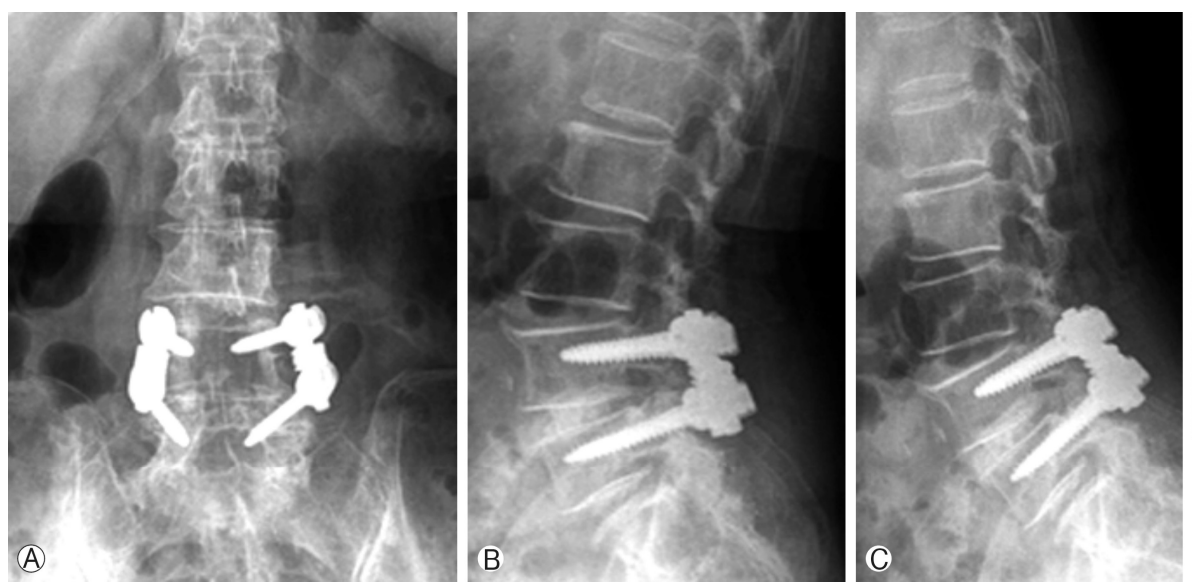

Fig. 3. (A) Radiographic evaluation or adjacent segment sagittal angle, and (B-D) postoperative fusion status. 

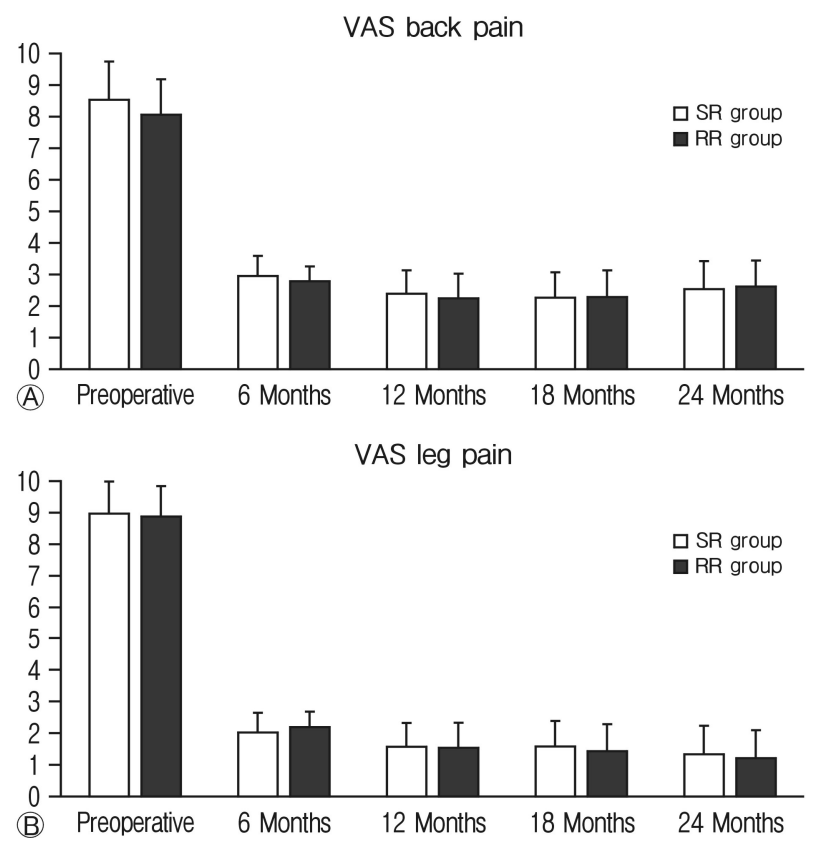

Fig. 4. Postoperative visual analog score (VAS) changes for back pain (A) and leg pain (B). SR, semirigid rod; RR, rigid rod.
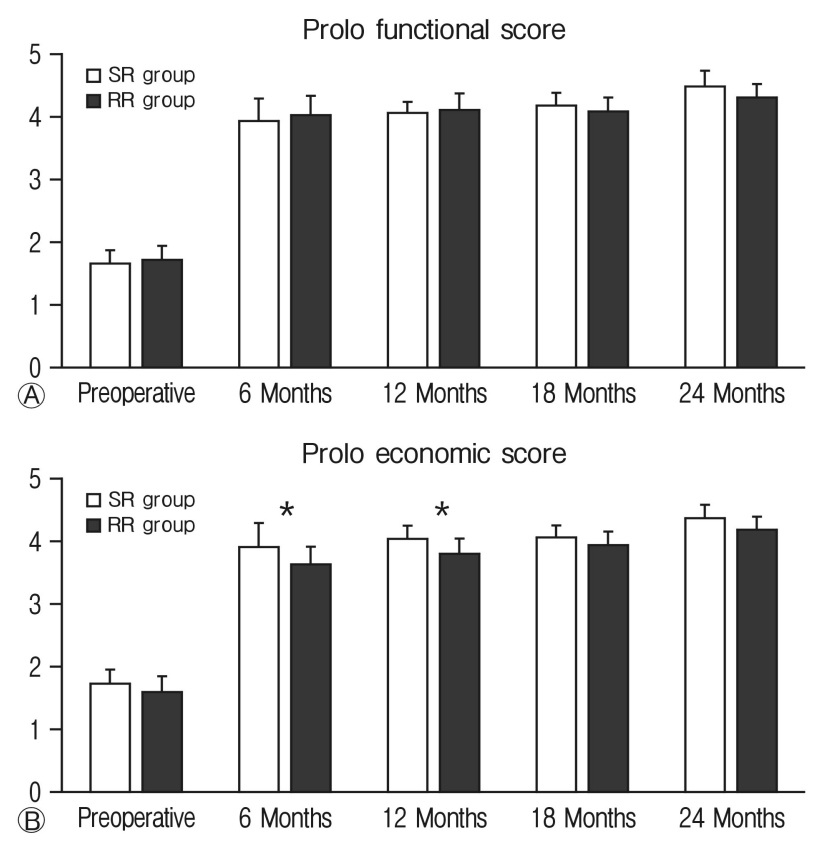

Fig. 5. Postoperative changes for functional (A) and economic (B) Prolo score. SR, semirigid rod; RR, rigid rod. " $\mathrm{p}<0.05$, statistically significant differences between the 2 groups.

and 2.20 in postoperative 6-month, 1.56 and 1.53 in 12-month, 1.56 and 1.43 in 18-month, and 1.33 and 1.23 in 24-month follow-up (Fig. 4B). There are no statistical differences in VAS-L were not observed between the 2 groups during 24 months follow-up. Mean PFS in SR and RR groups were 1.65 and
Table 2. Fusion rates after postoperative follow-up between the 2 groups

\begin{tabular}{lccc}
\hline \hline Follow-up & SR group & RR group & p-value \\
\hline Postoperative 6 months & $14 / 20(70.0)$ & $13 / 20(65.0)$ & 0.744 \\
Postoperative 12 months & $16 / 20(80.0)$ & $15 / 20(75.0)$ & 0.723 \\
Postoperative 18 months & $17 / 19(89.5)$ & $16 / 18(88.9)$ & 0.942 \\
Postoperative 24 months & $15 / 17(94.1)$ & $15 / 17(94.1)$ & 1.000 \\
\hline
\end{tabular}

Values are presented as number (\%).

$\mathrm{SR}$, semirigid rod; RR, rigid rod.

1.72 in preoperative status, 3.95 and 4.04 in postoperative 6-month, 4.05 and 4.12 in 12-month, 4.18 and 4.10 in 18month, and 4.50 and 4.33 in 24-month follow-up (Fig. 5A). There are no statistical differences in PFS were not observed between the 2 groups during 24-month follow-up. Mean PES in SR and RR groups were 1.72 and 1.60 in preoperative status, 3.89 and 3.60 in postoperative 6-month, 4.02 and 3.77 in 12-month, 4.03 and 3.90 in 18-month, and 4.33 and 4.17 in 24-month follow-up (Fig. 5B). There are no statistical differences in PES between the 2 groups at preoperative, but, PFS improved significantly in SR group compared to RR group at 6 and 12 months postoperatively $(\mathrm{p}<0.050)$. But, this advantage also become statistically not differently observed at 18 and 24 months pos- toperatively.

Radiological overall fusion rate in SR and RR groups were $70.0 \%$ and $65.0 \%$ at the 6-month follow-up, $80.0 \%$ and 75.0 $\%$ at the 12 -month follow-up, $89.5 \%$ and $88.9 \%$ at the 18 month follow-up, and $94.1 \%$ and $94.1 \%$ at the 24-month follow-up (Table 2). Fusion rates of SR group was slightly higher than RR group during all follow-up periods, but no statistical differences in fusion rate were observed between the 2 groups during 24-month follow-up. The accurate time points of fusion for the 2 groups were not investigated. Neither screw displacement nor screw failure was detected for any of the patients at the follow-up. No semirigid and rigid rod failure was found in both groups. In both groups, no new adjacent segmental degeneration was found. No surgical site infection or severe wound healing disorder was encountered.

\section{DISCUSSION}

Posterior lumbar fixation instrumentation is a surgical method for degenerative lumbar diseases, $3,13,15,16,19,24)$, and is hypothesized that it is the reduction in segmental motion by partial fusion, or by an alteration of the structure of the spinal tissues that results in the alleviation of pain ${ }^{11)}$. Rigid rods such as titanium and other titanium alloys had been preferred biomaterial for lumbar spinal fusion instruments because of their better strength, fatigue resistance, and biocompatibility compared to other metals ${ }^{20)}$. But, the rigid rods are probably far more rigid than is needed to promote fusion, and may lead to adjacent segment degeneration in patients, ${ }^{2,6,79}$. Another disadvantage of rigid rods is stress shielding, reducing the me- 
chanical load to the bone grafts in the intervertebral space, which can delay fusion and cause an abnormal load distribution that may result in implant failure ${ }^{1,10,21)}$. For these reasons, some authors presented the concept of semirigid fixation, which prompted the development of a spinal implant material with an optimum stiffness that could promote fusion while lowering the incidence of adjacent segmentdegeneration ${ }^{1,21)}$. This closer approximation to the physiological stiffness of bone might allow anteroposterior column load sharing and a potentially increased likelihood of fusion through the creation of higher anterior forces and increased contact between the graft and endplate for greater bone remodeling ${ }^{21,23)}$. In addition, biomechanical testing has demonstrated that segments instrumented with semirigid rods more closely imitate the physiological loading forces of the adjacent segments, which may reduce the probability of adjacent segment disease ${ }^{23)}$.

In this study, the authors compared the radiological and clinical outcomes of patients undergoing lumbar fusion with semirigid rods (20 patients) versus rigid rods system (20 patients). Clinical scores were significantly improved until postoperative 24-month follow-up as compared with preoperative scores in both groups, with similar levels of improvement observed at the same time points postoperatively between the 2 groups. This result is similar to previous finite element model $^{14)}$ and clinical study semirigid rods (PEEK) and titanium alloy rods ${ }^{20)}$. In a finite element model study by Kim et al. ${ }^{14)}$, arthrodesis with rigid (titanium alloy rigid rods) and semirigid posterior fixation (Nitinol semirigid rods) caused greater increase in disc pressure and contact force at the adjacent segments than arthrodesis without posterior fixation, and the increase in disc pressure and contact force differed according to the rigidity of the posterior fixation. Posterior semirigid fixation also increased the workload of the adjacent segments, but the increase was smaller than that of posterior rigid fixation. As result in finite element study, it is thought that posterior semirigid fixation can preserve the advantages and compensate for the disadvantages of rigid fixation in lumbar arthrodesis. In a previous study comparing semirigid rods (PEEK) and titanium alloy rods ${ }^{20)}$, semirigid rods also provide a similar clinical outcome as titanium rods in the short term. Clinical VAS-B, VAS-L, and Japanese Orthopedic Association (JOA) scores were significantly improved until 1 year postoperatively as compared with preoperative scores in both groups, and semirigid group showed a similar extent of improvement in VAS-B, VAS-L, and JOA scores at the same time points postoperatively as patients in rigid group.

Posterior rigid fixation in lumbar arthrodesis could offer greater stabilization and a higher fusion rate. Overly rigid spinal instrumentation is known to carry the inherent danger of segment instability adjacent to the fused levels, as well as stress shielding and bone-implant interface failure, with cascading pseudarthrosis ${ }^{1,10,18,21)}$. Semirigid fixation combined with lumbar arthrodesis seems to be able to decrease the loading of adjacent segments when compared with rigid fixation, whic $\mathrm{h}$ prompted the development of a spinal implant material with an optimum stiffness that could promotefusion ${ }^{1,14,21)}$. This closer approximation to the physiological stiffness of bone might allow anteroposterior column load sharing and a potentially increased likelihood of fusion through the creation of higher anterior forces and increased contact between the graft and endplate for greater bone remodeling ${ }^{21,23}$. But, no studies are not yet approved this, including clinical studies on the fusion rate as well as in vivo biomechanical studies. In this recent result, fusion rates of SR group was slightly higher than RR group during all follow-up periods, but, no statistical differences in fusion rate were not observed between the 2 groups during 24-month follow-up. In another clinical study comparing semirigid rods (PEEK) and titanium alloy rods ${ }^{20)}$, the interbody fusion was achieved in all patients treated with semirigid rods, likely associated with the anterior column load sharing and intervertebral space self-compressing characteristics of semirigid rods. But, these study has small number of patients in each group, so future studies with larger numbers of patients with long follow-up are required to confirm the true advantages of semirigid rods in lumbar fusion.

In the literature, semirigid fixation showed various advantages and disadvantages. Semirigid rods could meet the requirement in keeping lumbar lordosis and disc space height, and it provides a similar radiological and clinical outcome as titanium rods in the short term ${ }^{20)}$. Posterior semirigid fixation showed smaller increased the stress load of the adjacent segments than that of posterior rigid fixation in finite element study $^{14)}$. Indeed, current study showed significantly improved PESs in SR group compared to RR until 12 months, but this improvement became similar after 18 months. No significant complication was not observed in the literatures. But, the patients that required spinal correction could not be performed with semirigid rods because its' flexibility ${ }^{20)}$. And the price of semirigid rods is higher than that of rigid rods at present, which does not meet the socioeconomic point of view ${ }^{20)}$.

Although semirigid fixation system showed favorable result in this study, the authors still do not believe that all patients with degenerative lumbar disease can be treated with semirigid rods and stress that patient selection is an important consideration when using the semirigid rod system. This study only included in the patients with single level lumbar disc herniation, lumbar stenosis, and low-grade degenerative lumbar spondylolisthesis. The patients that required spinal correction which could not be performed with semirigid rods were not included in this study.

There were some limitations in our study. We had a small number of patients in each group with relatively short-term follow-up. Future studies with larger number of patients and a longer-term follow-up are required to confirm the true advantages and disadvantages of semirigid rods in lumbar fusion. Indeed, although biomechanical tests have demonstrated that semirigid rods can reduce the probability of adjacent segmental disease, the long-term follow-up of the use of semirigid rods in preventing this complication should be investigated. 


\section{CONCLUSION}

Semirigid rods offer a similar or superior radiological and clinical efficacy compared to rigid rods during 24-month follow-up. Semirigid implant (WavefleX) deserves careful consideration because the posterior semirigid fixation is seemed to preserve the advantages and to compensate for the disadvantages of rigid fixation in lumbar fusion operation.

\section{CONFLCT OF INTEREST}

No potential conflict of interest relevant to this article was reported.

\section{REFERENCES}

1. Ahn YH, Chen WM, Lee KY, Park KW, Lee SJ: Comparison of the load-sharing characteristics between pedicle-based dynamic and rigid rod devices. Biomed Mater 3:044101, 2008

2. Akamaru T, Kawahara N, Tim Yoon S, Minamide A, Su Kim $\mathrm{K}$, Tomita $\mathrm{K}$, et al: Adjacent segment motion after a simulated lumbar fusion in different sagittal alignments: a biomechanical analysis. Spine (Phila Pa 1976) 28:1560-1566, 2003

3. Bono CM, Lee CK: Critical analysis of trends in fusion for degenerative disc disease over the past 20 years: influence of technique on fusion rate and clinical outcome. Spine (Phila Pa 1976) 29:455-463, 2004

4. Bridwell KH, Sedgewick TA, O'Brien MF, Lenke LG, Baldus $\mathrm{C}$ : The role of fusion and instrumentation in the treatment of degenerative spondylolisthesis with spinal stenosis. J Spinal Disord 6:461-472, 1993

5. Cheh G, Bridwell KH, Lenke LG, Buchowski JM, Daubs MD, Kim Y, et al: Adjacent segment disease followinglumbar/thoracolumbar fusion with pedicle screw instrumentation: a minimum 5-year follow-up. Spine (Phila Pa 1976) 32:2253-2257, 2007

6. Cho KS, Kang SG, Yoo DS, Huh PW, Kim DS, Lee SB, et al: Risk factors and surgical treatment for symptomatic adjacent segment degeneration after lumbar spine fusion. J Korean Neurosurg Soc 46:425-430, 2009

7. Chosa E, Goto K, Totoribe K, Tajima N: Analysis of the effect of lumbar spine fusion on the superior adjacent intervertebral disk in the presence of disk degeneration, using the three-dimensional finite element method. J Spinal Disord Tech 17:134-139, 2004

8. Fischgrund JS, Mackay M, Herkowitz HN, Brower R, Montgomery DM, Kurz LT: 1997 Volvo Award winner in clinical studies. Degenerative lumbar spondylolisthesis with spinal stenosis: a prospective, randomized study comparing decompressive laminectomy and arthrodesis with and without spinal instrumentation. Spine (Phila Pa 1976) 22:2807-2812, 1997

9. Ghiselli G, Wang JC, Bhatia NN, Hsu WK, Dawson EG: Adjacent segment degeneration in the lumbar spine. J Bone Joint Surg Am 86-A:1497-1503, 2004
10. Gornet MF, Chan FW, Coleman JC, Murrell B, Nockels RP, Taylor BA, et al: Biomechanical assessment of a PEEK rod system for semi-rigid fixation of lumbar fusion constructs. J Biomech Eng 133:081009, 2011

11. Grob D, Benini A, Junge A, Mannion AF: Clinical experience with the Dynesys semirigid fixation system for the lumbar spine: surgical and patient-oriented outcome in 50 cases after an average of 2 years. Spine (Phila Pa 1976) 30:324-331, 2005

12. Iguchi T, Kanemura A, Kasahara K, Sato K, Kurihara A, Yoshiya S, et al: Lumbar instability and clinical symptoms: which is the more critical factor for symptoms: sagittal translation or segment angulation? J Spinal Disord Tech 17:284-290, 2004

13. Kanayama M, Cunningham BW, Sefter JC, Goldstein JA, Stewart G, Kaneda K, et al: Does spinal instrumentation influence the healing process of posterolateral spinal fusion? An in vivo animal model. Spine (Phila Pa 1976) 24:1058-1065, 1999

14. Kim TY, Kang KT, Yoon DH, Shin HC, Kim KN, Yi S, et al: Effects of lumbar arthrodesis on adjacent segments: differences between surgical techniques. Spine (Phila Pa 1976) 37:14561462, 2012

15. Kotani Y, Cunningham BW, Cappuccino A, Kaneda K, McAfee PC: The role of spinal instrumentation in augmenting lumbar posterolateral fusion. Spine (Phila Pa 1976) 21:278-287, 1996

16. Oh CH, Ji GY, Jeon JK, Lee J, Yoon SH, Hyun DK: Slip reduction rate between minimal invasive and conventional unilateral transforaminal interbody fusion in patients with low-grade isthmic spondylolisthesis. Korean J Spine 10:232-236, 2013

17. Park P, Garton HJ, Gala VC, Hoff JT, McGillicuddy JE: Adjacent segment disease after lumbar or lumbosacral fusion: review of the literature. Spine (Phila Pa 1976) 29:1938-1944, 2004

18. Pfeiffer M, Deike B, Clausen JD, Wilke A, Griss P: A new semirigid implant for instrumentation of scoliosis: preliminary report. Eur Spine J 10:427-436, 2001

19. Polly DW Jr, Santos ER, Mehbod AA: Surgical treatment for the painful motion segment: matching technology with the indications: posterior lumbar fusion. Spine (Phila Pa 1976) 30(16 Suppl):S44-51, 2005

20. Qi L, Li M, Zhang S, Xue J, Si H: Comparative effectiveness of PEEK rods versus titanium alloy rods in lumbar fusion: a preliminary report. Acta Neurochir (Wien) 155:1187-1193, 2013

21. Smit TH, Müller R, van Dijk M, Wuisman PI: Changes in bone architecture during spinal fusion: three years follow-up and the role of cage stiffness. Spine (Phila Pa 1976) 28:1802-1808, 2003

22. Turner JA, Ersek M, Herron L, Haselkorn J, Kent D, Ciol MA, et al: Patient outcomes after lumbar spinal fusions. JAMA 268: 907-911, 1992

23. Turner JL, Paller DJ, Murrell CB: The mechanical effect of commercially pure titanium and polyetheretherketone rods on spinal implants at the operative and adjacent levels. Spine (Phila Pa 1976) 35:E1076-1082, 2010

24. Zhou ZJ, Zhao FD, Fang XQ, Zhao X, Fan SW: Meta-analysis of instrumented posterior interbody fusion versus instrumented posterolateral fusion in the lumbar spine. J Neurosurg Spine 15: 295-310, 2011 